\title{
Science INSIGHTSB
}

\section{A Multimodal Discourse Analysis of Two Logos about Lanzhou China Junbang He and Shunliang Shi}

\section{Science Insights 2014; 6(1):100-104}

Science Insights is published by The Bonoi Academy of Science \& Education, Winston-Salem, NC 27157, USA

Copyright (C) 2014 The Bonoi Academy of Science \& Education. All rights reserved.

ISSN: 2329-5856

The online version of this article, along with updated information and services, is located on the World Wide Web at:

www.bonoi.org

Permissions: Requests for permissions to reproduce figures, tables, or portions of articles originally published in Science Insights can be obtained via our Permission Application System, a service of the Copyright Clearance Center. If you cannot access to this system, you can request permission through toour Editorial Office. Once the online version of the published article for which permission is being requested is located, Request Permissions in the middle column of the Web page under Services. Further information about this process is available in the Permissions and Rights Question and Answer document.

Submission: Information about submission to Science Insights is online at: http://www.bonoi.org/node/74 


\title{
A Multimodal Discourse Analysis of Two Logos about Lanzhou China
}

\author{
Junbang He, ${ }^{*,}{ }^{\Delta}$ Shunliang Shi ${ }^{*}$
}

BACKGROUND Logos are typical multimodal discourses combining languages, pictures, colors and so on with spatial distribution and often used in propagandizing a city. Lanzhou, the provincial capital city of Gansu Province in north-west China, released two logos about the image and the tourism successively to propagandize the city.

OBJECTIVE The purpose of the study was to analyze the logos from the perspective of linguistics and to identify how images and words in the logos work together to fulfill the aim of propagandizing the city, what the respective advantages and disadvantages of the two logos are, and how to achieve a better propaganda aim of the city logos.

METHOD Based on the Kress and Van Leeuwen's visual grammar, this paper attempts to adopt a multimodal analysis and descriptive-analytical method to two logos about Lanzhou China and make a comparison between them, analyzing the representational meaning, the interactive meaning and the compositional meaning of the two logos. CONCLUSION We found that images and words can work together to create a synergic effect of propagandizing the city, and multimodality is an effective way to present such information about the characteristics of a city in meaningmaking process of logos. Through the interaction of these different semiotic elements, the logos express much more abundant information than separate ones.

\begin{abstract}
*: School of Foreign Languages, Lanzhou University of Technology, Lanzhou, China

$\Delta$ : Correspondence to: Dr. Junbang He, No. 287, Langongping Street, Qi Li He District, School of Foreign Languages, Lanzhou University of Technology, Lanzhou 730050, China. Tel: +86-139-1989-6045; Email: hejb@lut.cn
\end{abstract}

Submitted: 26 November 2013

Revised: 14 December 2013

Accepted: 14 December 2013

[PC]: Publisher Copyright Paper
- How to Cite This Paper: He J, Shi S. A multimodal discourse analysis of two logos about Lanzhou China. Science Insights 2014;6(1):100-104.

Keywords: Multimodal discourse analysis -Logos -Lanzhou

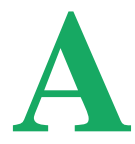
CCORDING to the World Tourism Organization, China will become one of the largest tourist destinations all over the world in the next decade. Therefore, it is crucial in tourism to deliver or present the information about tourism resources and unique cultures in China to domestic and foreign tourists and investors. Tourism is also one of the most important industries and has been increasingly developing in Lanzhou China, which attracts not only domestic tourists but a great number of foreign friends. Along with the de- 
velopment and the increasingly important status of tourism in the overall development, propagandizing the city in an effective way is becoming more and more important. Therefore, the local government released one vivid logo about the tourism of Lanzhou and another one about the general image of Lanzhou successively. The logo of a city is one of the most effective and common forms with very vivid and rich information which combines languages, number, pictures and so on with spatial distribution and is "the meaningful text realized by symbols by any more than one semiotic code"(1), that is , multimodal discourse.

Two books mark the birth of Multimodal Discourse Analysis (MDA): the Language of Displayed Art (7) and Reading Images (4). Kress and Van Leeuwen propose a comprehensive and systematic theoretical framework of visual grammar, providing theoretical foundation and methodology to understand and analyze multimodal discourses. Since Li (1) introduced Kress and Van Leeuwen's visual grammar to China, many articles have appeared in periodicals and magazines around China. $\mathrm{Hu}$ (3) and Zhu (1) have made comprehensive explanation to the theoretical foundation of MDA and its research methods as well as its application in social practices. In addition, a number of scholars apply MDA to diverse fields such as textbooks (2), advertisement (9), academic speech presentation (11), film or film poster (12), picture (10), news (13) and many other aspects of MDA. But there are only a few logo researches which adopt the perspective of visual grammar of multimodal discourse analysis, and most of the researches mainly involve logo of web page (7) or enterprise logo (13). This paper attempts to adopt Kress and van Leeuwen's methodology to briefly analyze two logos from Lanzhou, China and discuss how different modes work together to create a comprehensive meaning to fulfill the propaganda aim.

METHODS AND MATERIALS

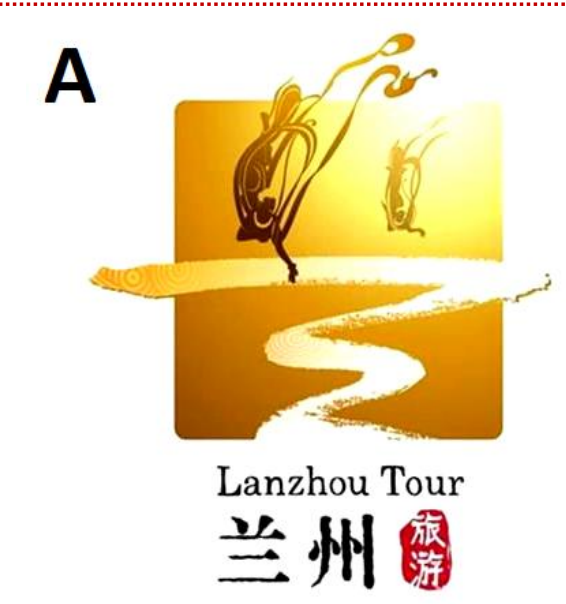

B

中国西北游 出发在兰州

Figure 1: The two logos used in this MDA modality. (A) The logo about Lanzhou tourism was released in 2012. Lanzhou is one of the most important cities on the ancient silk road and the unique provincial capital city in China through which the Yellow River is running, and "Dunhuang Feitian" is one of the major symbols of Gansu province even the northwest China. The images of the Yellow River in a zigzag form and the two flying "Dunhuang Feitian" in the logo form an ensemble image of the Chinese character "兰" (Lan). On the images of the Yellow River, there is some typical design of Ma Jia Yao culture representing the ancient booming culture of Yellow River watershed, and the color of the background represents the loess plateau of the North-west China. The lingual parts include two points: Lanzhou Tour; Lanzhou as the Center of Tour in Northwest China. Available from: http://www.linbang0769.com/60/n-256.html. Last accessed date: December 14, 2013. (B) The logo about the image of Lanzhou city was released in 2013. The Yellow River Mother Sculpture is one of the most typical and representative symbols of Lanzhou city, and its image is the center of the logo; the design of Ma Jia Yao culture gives prominence to the characteristics of the culture of Yellow River watershed; the three stripes (in blue, orange and green respectively) symbolize the blue sky, the Yellow River and the loess plateau, and the green mountains of Lanzhou region respectively. The lingual parts means Lanzhou China is the city of the Yellow River. Available from:

http://www.gs.xinhuanet.com/news/2013-05/24/c_115888461.htm. Last accessed date: December 14, 2013.

\section{Multimodal Discourse Analysis}

The aim of MDA is to take representational, interactive and compositional meanings labeled in the various communicative modes into consideration to analyze how they work together to create a complete discourse or communicative event.

1. Representation can either be narrative, presenting unfolding actions and events, processes of change, transitory spatial arrangements, or conceptual, representing participants in terms of their class, structure or meaning.
2. Interactive meaning is about the relationships among the three components of an image: the designer, the participant, and the viewer. It can be realized by four major factors: contact, distance, attitude and modality, which can work together to create complex and subtle relations between the viewer and the participant.

3. Composition relates the representational and interactive meanings of the image to each other through three interrelated systems: information value, salience, and framing. (4) 
The MDA of visual grammar provides theoretical foundation and study method for the present modality analysis, in which a descriptive-analytical method is adopted.

\section{DISCUSSION}

To conduct propaganda of Lanzhou tourism and the city, the local administration adopts various approaches including the above vivid logos, which present the typical information in a general way to help viewers instantly know the general characteristics, charm and representatives of the city.

\section{Representational Meaning}

In the representational analysis, Kress and van Leeuwen draw into the concept of "vector" to distinguish narrative and conceptual representations. In the narrative pattern, participants are connected by a vector or vectors and represented as doing something to or for each other, while the conceptual pattern represents participants in terms of their generalized, more or less stable and timeless essence (4).

In the first logo, the typical images include the Yellow River in a zigzag form and two "Dunhuang Feitian" constituting an ensemble image of the Chinese character “兰”. One Feitian's arm and the Yellow River intercross to form the most obvious vector, and the ribbons of Feitian also form vectors. The vectors indicate a narrative representation, which presents unfolding actions or events about the city tourism and transitory spatial arrangements. It could be understood as an invitation to this wonderful city through which the Yellow River is running and as an implication that there is something amazing and unique in this region of the loess plateau. At the same time, the ensemble image “兰”, the Chinese characters “兰州”, the seal with the characters “旅游” and the English words "Lanzhou" and "tour" distinguish the logo as a conceptual representation as well. In addition, there are other conceptual elements such as the flowing Yellow
River with the typical design of Ma Jia Yao culture representing the ancient booming culture of Yellow River watershed, the flying Feitian with fluttering ribbons and the bright yellow background representing the loess plateau. All these elements make up the main portion of the whole logo and interconnect with each other in a classificatory, analytical and symbolic way, making the logo full of dynamics, hope, enthusiasm and delight and highlighting the exact topic and meaning of the logo. Therefore, the logo is very typical and appropriate to present the information of Lanzhou city and tourism in northwest China.

The second logo is conceptual without vectors. The main images of concept include the Yellow River, blue sky, green land, the words in both languages and the Yellow River Mother Sculpture. There also exists an ensemble Chinese character “兰”. Three aspects of the conceptual meaning are involved - classificatory: all the above elements are subordinate to the concept "Lanzhou City" or "Lanzhou Tourism"; analytical: each of the elements is the possessive attribute, and the ensemble image “兰”, the character “兰州” and the words "Lanzhou" are the carriers; symbolical: the Yellow River Mother Sculpture is the center of the logo and one of the most typical and representative symbols of the city, the design of Ma Jia Yao culture gives prominence to the characteristics of the culture of Yellow River watershed, the blue sky and green land show that excellent environment is always the beautiful dream that local people have been trying to fulfill with unremitting efforts, and the three stripes (in blue, orange and green respectively) symbolize the blue sky, the Yellow River and the loess plateau, and the green mountains of Lanzhou respectively. So this logo also shows the landforms and the characteristics of the city and fulfills the goal of propagandizing effectively.

\section{Interactive Meaning}

Contact
When the participant looks directly at the viewer, or there are eye contacts between them, it is "demand" image; when there is no eye contact, "offer" image (4).

In both logos, the participant is not a living human but quasi-human, there is no eye contact between the participant and potential viewer, and it is taken as an imaginary "contact", that is, personal relationship between the participant and the viewer does not exist. These images are "offer", only expressing the meaning of information that provides potential viewer with very general and brief but representative characteristics, symbols and other information about Lanzhou. The verbal mode also "offers" the similar information.

\section{Distance}

There are six types of distance between the participant and the viewer: intimate distance, close personal distance, long personal distance, close social distance, long social distance (the whole person shown, and surrounded by space) and public social distance. The different types are the standards for judging the distance between the participant and the viewer in an image (4).

In the logos, the whole bodies of Feitian and the Yellow River Mother Sculpture are shown and surrounded by space, reflecting the long social distance between the participant and the viewer, which indicates that the viewer need to learn about this city better and more thoroughly. It is the case especially for nonlocals, for many of them virtually have some misunderstandings and wrong ideas about the city. Attracted by the logos and the transmitted information, people would like to visit Lanzhou and northwest China, which is the propaganda aim of the logos. Even some local people do not know and understand the city, its culture and its characteristics well, so they could be also attracted by the logos and would like to learn more about their city.

12 JANUARY 2014 SCIENCE INSIGHTS VOL. 6, NO. 1 www.bonoi.org 
Attitude is used to analyze the representation of a subjective image, and perspective is the way in which images bring about relationships between represented participant and the viewer. Horizontal perspective includes frontal angle which makes the viewer feel that he or she is experiencing the image personally or feels what the participant feels and oblique angle which conveys a message that the viewer as a bystander holds an indifferent and unconcerned attitude. On the other hand, vertical perspective involves a view angle of looking down, which represents a forceful position the viewer stands, a view angle of looking up, which shows a forceful position of the participant, and a straight angle which suggests a relatively equal relationship between the participant and the viewer (4).

From horizontal perspective, both of the logos have a frontal angle which shows involvement, that is, the viewer is "involved" with the represented participants, what the viewer sees in the logos is part of the world we are involved in, the viewer could feel that he or she is experiencing the image personally or feels what the participant feels, and the viewer could enjoy and appreciate the beautiful scenery and culture such as the Yellow River running through the city, the characteristics of the landforms, and rich culture represented in Feitian and so on when visiting Lanzhou or being in the region.

From vertical perspective, two logos show different interactive meanings. In the first logo, the viewer has a view angle of looking up at the represented participants Feitian, which shows a forceful position of the participant over the viewer. Feitian, flying in the sky and serving or praising the Buddha and other gods, do not belong to earthly world and have a forceful position in the aspect of art, culture or religion. When visitors go to Dunhuang, they would also look up at the Feitian in the murals on the wall or vault. In addition, the Yellow River is at eye level, which is a straight angle and suggests a relatively equal relationship, that is, the river running through the city is very close and common to people. In the second logo, the sculpture is at eye level and the viewer has a straight angle, which suggests a relatively equal relationship between them. When visiting the city and appreciating the Yellow River Mother Sculpture, which is one of the landmarks of the city and connotes that the Yellow River has nourished generations of Chinese people, visitors would like to look at it closely and take photos with it, and this shows equal relationship between the participant and the viewer.

\section{Modality}

There are eight markers of visual modality, color saturation, color differentiation, color modulation, contextualization, representation, depth, illumination and brightness. Each of these dimensions can be seen as a scale of high, medium or low sensory modality (4).

For the first logo, the hue of image and the background from brown to yellowish is lowly saturated, the black words and characters are of lowest saturation, and the red seal is of high saturation; the color differentiation is not very obvious, but the degree of color modulation is high. For the second logo, the bright colors of blue, yellow and green make it highly saturated, while the white image of the Yellow River Mother Sculpture, the black words and the characters are absent of color; the color differentiation is very obvious with high degree of color modulation. On the other hand, the logos have medium contextualization scale with an articulated but not very detailed background of the Yellow River, the yellow land and the sky in bright light. The detail of representation is reduced and abstracted to much lesser and coarse degree, and the texture is also omitted altogether - the Yellow River, Feitian and the Sculpture are then represented merely by the lines that trace the contour with plain flat mono-color, and other elements are presented just by stripes in different colors in the second logo. As for depth, the first logo, with the zigzag Yellow River below, the bigger Feitian above and the smaller Feitian in the distance is perspective and has comparatively high modality, while the second logo is not perspective. As for illumination, there is very low scale of shadows and light in the first logo (lowly illuminated), and there is not any in the second one (abstracted from illumination). Finally, brightness values can also contrast to a greater or lesser degree: in the first logo, there is a range of yellow hue from dark brown to yellowish even pale yellow, which is of high scale of brightness; in the second logo, there is no scale of brightness. Above all, the logos have medium sensory modality generally, but the modality of the first one is much higher than the second one.

\section{Compositional Meaning Analy- sis}

\section{Information Value}

Information value refers to the placement of elements that endows them with the specific informational values attached to the various "zones" of the image: left and right, top and bottom (the ideal and the real), center and margin (nucleus and subservience) (4).

In the first logo, Feitian is placed on the top as the ideal, and the image of Yellow River is placed at the bottom as the real. The character "Lanzhou Tour" and the ensemble image of “兰” are presented in the center as the nucleus of the information; other elements are in some sense subservient. In the second logo, the Sculpture and the characters “中国兰州” are presented in the center as the nucleus of the propaganda, while other elements are presented as margin or background. In both logos, there are similar valuation between the image and the verbal mode to present the similar valuation.

\section{Salience}

Salience is realized by such factors as placement in the foreground or background, relative size, contrasts in tonal value (or color), differences in sharpness, etc. to attract the viewer's attention to different degrees (4). 
In the first logo, the image of Feitian, the river, the ensemble image “兰”, the big character “兰州” and the red seal “旅游” are most salient with big size, sharpness of focus and obvious color contrasts. Both of the two elements Feitian and the Yellow River placed in the foreground are potent cultural and regional symbols and representative. In the second logo, the Sculpture and the characters “中国兰 州” are placed in the foreground with relative big size and most salient. As for the general salience on the page, the image is of higher salience with more than half percentage of the logos. These most salient elements are the most eye-catching in the composition, not just because they are placed in the foreground and form the biggest elements in the pictures but also because they are in sharper focus and present the major topic and meaning of the logos.

\section{Framing}

Framing refers to the presence or absence of framing devices (realized by elements which create dividing lines, or by actual frame lines) that disconnect or connect elements of the image, signifying that they belong or do not belong together in some sense (4).

In the first logo, there is mediadegree-connection between the image elements. Two zigzag lines that form the edges of the Yellow River run through the middle of the picture, and one line is also crossed with one Feitian's arm to show the connection. The blurred line between the image part and the verbal part also show the close connection, and the word "在" and "Lanzhou Tour" are connected closely by the image. The image of
"Feitian", the Yellow River, and the word "Lanzhou" makes up the major framing of elements. In the second logo, three stripes in different colors form lines, broken lines or shared lines to show not only the differences of the elements but also the connections between them, thus creating a comprehensive meaning of the logos.

\section{CONCLUSION}

The two logos about Lanzhou are composed of different modes such as vivid visuals which are the symbols or representatives of the city and very brief verbals which focus on the topic and the propaganda to correspond to the characteristics and ultimate goals of propaganda of logos. The multimodal discourse analysis of logos overcomes the limitations of monolingual methods which ignores the interaction of different semiotic modes and provides a more effective way to create and understand the meaning of such city logos. According to the analysis, we could see multimodality is an effective way to present information about the characteristics of a city in meaning-making process of logos. Through the interaction of these different semiotic elements, the logos express much more abundant information than separate ones. In addition, based on the above analysis, we could also see the first logo is relatively more vivid, informative and dynamic than the second one.

\section{Author Contributions}

The paper is written by Junbang He. Professor Shunliang Shi is the tutor who offers very earnest tutorial instruction in the work.

\section{Abbreviations}

MDA: Multimodal Discourse Analysis

\section{Conflict of Interests}

None

\section{References}

1. Li Z. Social semiotic approach to multimodal discourse. Foreign Lang Res 2003; 5:1-8.

2. O'Toole. M. The Language of displayed art. London: Leicester University Press, 1994.

3. Kress G.T. van Leeuwen T. Reading Images: the grammar of visual design. London: Routledge, 1996.

4. Hu Z. Multimodalization in social semiotics. Lang Teach Ling Stud 2007;1:110.

5. Zhu Y. Theory and methodology of multimodal discourse analysis. Foreign Lang Res 2007; 5:82-6.

6. Chen $\mathrm{Y}$, Wang $\mathrm{H}$. Ideational meaning of image and text-image relations. $J$ Ningbo Univ (Education Edition) 2008;1:124-9.

7. Zhang W. Multimodal discourse analysis from the perspective of social semiotics. J Northeast Norm Univ (Philos Soc Sci) 2012;2:224-5.

8. Hu J, Zeng L. Constructing semiotics in multimodel discourse: a study of academic speech presentation. Foreign Lang Their Teach 2007;3:12-5.

9. Qiao Y. A multimodal analysis to a movie poster-"Slumdog Millionaire", the Biggest Winner at 81st Oscar. Literatures (Lang Res) 2010; 8:144-6.

10. Zeng F. A Study of image-text relations and their patterns in multimodal text:comments on three theories of image-text relations. J Sichuan Int Stud Univ 2010;4:60-4.

11. Wang J. A case study of visual semiosis by multimodal critical discourse analysis. J Int Commun 2012;4:51-6.

12. Wang L. A Study about a baidu logo based on multimodal discourse analysis. J Panzhihua Univ 2011;28:76-8.

13. Zhang S. A comparative multimodal analysis of a foreign enterprise logo and a domestic enterprise logo. North Liter 2010;7:96-7. 